\title{
Single and double layer centrifugation improve the quality of cryopreserved bovine sperm from poor quality ejaculates
}

\author{
Alessia Gloria ${ }^{1 *}$, Augusto Carluccio ${ }^{2}$, Laura Wegher ${ }^{2}$, Domenico Robbe ${ }^{1}$, Giovanni Befacchia ${ }^{3}$ and Alberto Contri ${ }^{1}$
}

\begin{abstract}
Background: Density gradient centrifugation was reported as a technique of semen preparation in assisted reproductive techniques in humans and animals. This technique was found to be efficient in improving semen quality after harmful techniques such as cryopreservation. Recently a modified technique, single layer centrifugation, was proposed as a technique providing a large amount of high quality spermatozoa, and this treatment was performed before conservation. Single layer centrifugation has been studied prevalently in stallions and in boars, but limited data were available for bulls. Occasionally bulls are known to experience a transient reduction in semen quality, thus techniques that allow improvement in semen quality could be applied in this context. The aim of this study was the evaluation of single layer and double layer centrifugation by the use of iodixanol, compared with conventional centrifugation and non-centrifuged semen, on the sperm characteristics during the cryopreservation process in bulls with normal and poor semen quality.

Results: Single layer centrifugation and double layer centrifugation both significantly increased the percentage of normal spermatozoa and decreased the percentage of non-sperm cells in poor quality samples, while both were ineffective in those of normal quality. Sperm characteristics in poor quality samples increased after single layer centrifugation and double layer centrifugation, reaching values similar to those recorded in normal samples, and this trend is maintained after equilibration and after cryopreservation. On the other hand, SLC and DLC resulted in a consistent reduction in the spermatozoa recovered, and this resulted in a reduction of the absolute amount of spermatozoa cryopreserved in the normal samples, without a clear improvement in sperm characteristics in this type of sample.

Conclusions: These data suggested that both SLC and DLC could be performed in practice, but their application should be limited to the cases in which the quality of the spermatozoa recovered is more important than the total amount of spermatozoa.
\end{abstract}

Keywords: Bull, Cryopreservation, Density gradient centrifugation, lodixanol, Semen analysis

\section{Background}

Artificial insemination (AI) is generally considered to be the technique that has contributed the most to the improvement of animal reproduction. This technique can result in pregnancies only if the insemination dose contains sufficient viable spermatozoa capable of reaching the site of fertilization [1]. In this context, semen

\footnotetext{
* Correspondence: gloriaalessia@libero.it

'Faculty of Veterinary Medicine, University of Teramo, Località Piano D'Accio, 64100 Teramo, Italy

Full list of author information is available at the end of the article
}

analysis is the most commonly used procedure to evaluate male fertility potential in humans and animals [2-5].

Density gradient centrifugation through colloids is one of the procedures recommended by the World Health Organization for the preparation of human spermatozoa used in assisted reproduction [6]. In animals, this method has been suggested as a potential means of improving the quality of sperm after cryopreservation [7-9]. Recently, a modified gradient centrifugation technique, single layer centrifugation (SLC), was proposed to improve the semen quality before conservation and AI $[1,10]$. This technique provides high 
quality sperm, with an increased number of motile and membrane-intact spermatozoa. On the other hand, a significant loss of spermatozoa during the centrifugation procedure was reported $[1,11]$, and the absolute number of efficient sperm recovered after this procedure was lower than in conventional centrifugation [12]. This procedure should therefore be reserved for cases in which the sperm characteristics after density centrifugation are more important than the total amount of spermatozoa recovered. Evidence has suggested that the recovery rate of spermatozoa after centrifugation is related to the g-force applied. On the other hand, an excessive centrifugation force reduced the viability of spermatozoa, probably because of the physical pressure against the wall of the tube [13-15].

In a study performed on bulls, SLC was found to increase sperm mitochondrial membrane potential, reactive oxygen species (ROS) production, and protein phosphorylation, and to decrease sperm chromatin damage, while other parameters such as sperm motility, membrane integrity and morphology remained similar [16]. However, these effects were recorded soon after centrifugation, but no data on the long term effects or the impact of this procedure on cryopreservation were verified.

The primary aim of this study was the evaluation of the effect of SLC and DLC on morphological and functional quality of fresh spermatozoa in bulls with normal and poor semen quality. Secondly, the effect of centrifugation (SLC, DLC, and conventional centrifugation) on bovine semen characteristics after equilibration and cryopreservation was evaluated.

\section{Methods}

\section{Animals and preliminary semen evaluation}

This study was performed on 20 Swiss Brown bulls belonging to Superbrown Consorzium Bz/Tn (1.5 to $5 \mathrm{yr}$ old). The bulls were housed in the Alpenseme AI Center of the Provincial Breeders Federation of Trento (Ton, Trento, Italy). Animals used in this study were managed according to the National Law for Animal Welfare and Protection (Italy).

Semen was collected using an artificial vagina and evaluated. The volume was measured from the graded collection tube soon after collection; concentration was determined using Accucell photometer (IMV Technologies, L'Aigle, France) after dilution 1:100 with saline solution. Progressive motility (PM) was evaluated subjectively (magnification: $\times 400$ ) at $37^{\circ} \mathrm{C}$ using phase contrast microscopy, after dilution with prewarmed Bioxcell (IMV Technologies) at $80 \times 10^{6}$ sperm $/ \mathrm{mL}$. Morphology was evaluated after dilution at $80 \times 10^{6}$ sperm $/ \mathrm{mL}$ with $0.9 \% \mathrm{NaCl}$ solution and $3 \%$ glutharaldehyde using phase contrast microscopy (magnification: $\times 1,000)[17,18]$.
Bulls were classified with normal semen quality $(\mathrm{N})$, with subjective progressive motility $\geq 70 \%$ and normal sperm morphology $\geq 70 \%$, or poor semen quality $(\mathrm{P})$, with PM and morphology lower than the thresholds. Part of the ejaculate for each bull was used for the study.

\section{Centrifugation procedures}

To evaluate the effect of SLC and DLC on sperm characteristics after centrifugation, ejaculates were divided into four aliquots and three of them were diluted with a modified Tyrode's medium (TALP) [19] at $30 \times 10^{6}$ sperm $/ \mathrm{mL}$. The fourth (Control non-centrifuged - NC) sample was diluted at $30 \times 10^{6} \mathrm{sperm} / \mathrm{mL}$ with Bioxcell (IMV Technologies) and was processed without centrifugation.

The Optiprep (Axis-shield, Olso, Norway), as iodixanol $60 \%$, was diluted with TALP to reach a concentration of $15 \%$ iodixanol (top layer - $1.075 \mathrm{~g} / \mathrm{mL}, \mathrm{pH}$ 7.4, $302 \mathrm{mOsm}$ ) and of $35 \%$ iodixanol (bottom layer $1.192 \mathrm{~g} / \mathrm{mL}, \mathrm{pH}$ 7.3, $298 \mathrm{mOsm})$.

For SLC, $4 \mathrm{~mL}$ extended semen were layered over $4 \mathrm{~mL}$ of iodixanol $15 \%$ in a $15 \mathrm{~mL}$ Falcon tube, taking care not to allow the semen to mix with the colloid. After centrifugation at $1,000 \times \mathrm{g}$ for 20 mins, the supernatant (seminal plasma plus extender) and most of the colloid were discarded. The sperm pellet was resuspended at the original volume with Bioxcell (IMV Technologies).

For DLC, $4 \mathrm{~mL}$ of iodixanol $15 \%$ was placed first, then $4 \mathrm{~mL}$ of iodixanol $35 \%$ was gently deposited at the bottom of the tube; finally, $4 \mathrm{~mL}$ of extended semen were layered on the top of the iodixanol solutions. After centrifugation at $1,000 \times \mathrm{g}$ for 20 mins, supernatant and the bottom $35 \%$ of iodixanol were removed and sperm pellet was resuspended at the original volume with Bioxcell (IMV Technologies).

Conventional centrifugation (CC) was performed by the centrifugation of a $4 \mathrm{~mL}$ aliquot of diluted semen at $600 \times \mathrm{g}$ for $10 \mathrm{mins}$ without colloid, and then resuspended at the original volume with Bioxcell CSS I and II (IMV Technologies).

After processing, sperm characteristics were evaluated in all samples.

\section{Cryopreservation}

All four samples from each bull were transferred to a beaker containing water at room temperature, then were cooled passively to $5{ }^{\circ} \mathrm{C}$ for $1 \mathrm{~h}$. Samples were equilibrated at the same temperature for an additional $2 \mathrm{~h}$. At the end of the equilibration period, one aliquot from each sample was evaluated. The equilibrated samples were loaded in $0.25 \mathrm{~mL}$ French straws and frozen in a programmable nitrogen vapor freezer (DigitCool, IMV Technologies), using a conventional freezing rate [20]. 
The straws were then plugged into liquid nitrogen and stored for at least $7 \mathrm{~d}$. Ten straws for each treatment were thawed at $37{ }^{\circ} \mathrm{C}$ for $60 \mathrm{~s}$ in a waterbath, incubated for a further 10 mins at $37{ }^{\circ} \mathrm{C}$ and then evaluated.

\section{Semen evaluation}

The evaluation of the sperm kinetics, morphology, viability/acrosome integrity and mitochondrial membrane potential were performed on samples after centrifugation, equilibration, and cryopreseration.

\section{Recovery rate}

The recovery rate was calculated, measuring sperm concentration by a Burker counting chamber before and after the centrifugation, as follows:

(concentration after centrifugation/concentration before centrifugation) $* 100$.

\section{Sperm morphology}

In each sample spermatozoa were evaluated and classified as previously reported in the bull [21], and the percentage of each abnormality, such as the total sperm abnormality, was calculated on at least 200 spermatozoa. The percentage of non-sperm cells was also estimated and recorded in samples. Epithelial cells, leukocytes and round cells were classified as non-sperm cells.

\section{Sperm kinematic evaluation}

Objective sperm kinetic was measured with a computer assisted sperm analyzer (CASA) IVOS 12.3 (HamiltonThorne Bioscience, Beverly, MA, USA). An aliquot of each sample was rewarmed at $37^{\circ} \mathrm{C}$ for 10 mins and analyzed in a Makler chamber (Sefi Medical Instruments, Haifa, Israel), as previously reported [22]. In this study, the following parameters were considered: total motility (TM, \%), progressive motility (PM, \%), average path velocity (VAP, $\mu \mathrm{m} / \mathrm{s})$, straight line velocity (VSL, $\mu \mathrm{m} / \mathrm{s}$ ), curvilinear velocity (VCL, $\mu \mathrm{m} / \mathrm{s})$, amplitude of lateral head displacement $(\mathrm{ALH}, \mu \mathrm{m})$, beat cross frequency (BCF, Hz), straightness (STR, as VSL/VAP, \%), and linearity (LIN, as VSL/VCL, \%). Spermatozoa with a VAP $\geq$ $80 \mu \mathrm{m} / \mathrm{s}$ and STR $\geq 75 \%$ were considered progressive [17]. The CASA settings used in this study were those previously reported [23].

\section{Sperm membrane and acrosome integrity}

The sperm membrane and acrosome integrity were evaluated simultaneously by flow cytometry. Samples $(1 \mathrm{~mL})$ were stained with $2.4 \mu \mathrm{mol}$ of propidium iodide (PI) and $5 \mu \mathrm{g} / \mathrm{mL}$ of FITC-conjugated agglutinin derived from pisum sativum (FITC-PSA). After 10 mins of dark incubation at room temperature, each sample was analyzed by the flow cytometer EPICS XL (Beckman Coulter, San Jose, CA, USA). Acquisitions were conducted using the System II software (Beckman Coulter, USA). The samples were excited with a 20-mW argon ion 488-nm laser. The FITC-PSA fluorescence was collected in the FL1 sensor using a 530/28 nm band-pass, while the PI fluorescence was obtained using the FL3 sensor through a $660 / 20 \mathrm{~nm}$ long pass filter. Forward and side-scatter values were recorded on a linear scale and fluorescence values on a logarithmic scale. No compensation was used between the fluorescent channels. The flow cytometric analysis was performed at a flow rate of 6 to $24 \mu \mathrm{L} / \mathrm{min}$, and the acquisitions were stopped at 30,000 events. This stain association resulted in four different subpopulations: sperm with membrane integrity and acrosome integrity showing no fluorescence (PI-/PSA-); sperm with membrane integrity and an acrosome reaction (PI-/PSA+); sperm with a damaged membrane and acrosome integrity (PI+/PSA-); and sperm with a damaged membrane and a reacted acrosome (PI+/PSA + ).

\section{Mitochondrial membrane potential analysis}

Mitochondrial membrane potential (MMP) of spermatozoa was evaluated using the fluorescent stain 5,5 6,6 -tetrachloro-1,1 ,3,3 -tetraethyl-benzimida zolylcarbocyanine chloride (JC-1). The lipophilic cationic fluorescent carbocyanine dye, JC-1, was used to differentially label mitochondria with high and low membrane potential [24]. The sperm suspension was adjusted to a density of $5 \times 10^{6} \mathrm{sperm} / \mathrm{mL}$ and incubated for $45 \mathrm{mins}$ at $37{ }^{\circ} \mathrm{C}$ in the dark with $\mathrm{JC}-1$ probe $(5 \mu \mathrm{mol})$. At the end of the incubation period, cells were washed in TALP and evaluated using the flow cytometer EPICS XL (Beckman Coulter) equipped with the System II software (Beckman Coulter) as previously reported [25]. The green fluorescent emissions of the monomeric form of JC-1 (mitochondria with low potential - IMMP) were collected by a $530 \pm 15-\mathrm{nm}$ filter (FL 1), and the orange emission of the polymeric form of JC-1 (mitochondria with high membrane potential - hMMP) by a $585 \pm 21-\mathrm{nm}$ filter (FL 2). The flow cytometric analysis was performed at a flow rate of 8 to $30 \mu \mathrm{L} / \mathrm{min}$, and the acquisitions were stopped at 30,000 events. No compensation was used between the fluorescent channels.

\section{Statistical analysis}

The data are presented as mean \pm standard deviations (SD). Sperm characteristics for each treatment (SLC, DLC, CC and NC) at each evaluation point (before centrifugation, after centrifugation, after equilibration and after cryopreservation) in each bull group (normal and poor semen quality), were compared using a general linear model (GLM) based on the univariate ANOVA. The post-hoc analysis was performed using the Scheffè test. 
The bull was included as a random factor, in order to verify a possible effect on the results. In all cases, the differences were considered to be significant with $\mathrm{P} \leq 0.05$.

The statistical analyses were performed using the SPSS 17.0 software package (SPSS Inc. Chicago, IL, USA).

\section{Results}

Raw semen characteristics from bulls with normal and poor semen quality involved in this study are summarized in Table 1. Sperm total and progressive motility, sperm velocities, and sperm morphology were significantly different in samples with normal and poor semen quality $(P \leq 0.05)$.

Single layer centrifugation and DLC showed a reduced recovery rate in all samples. The mean recovery rate was $56 \pm 11 \%$ for SLC and $44 \pm 14 \%$ for DLC. No significant differences were found between the two methods. The $C C$ showed a higher $(P \leq 0.05)$ recovery rate $(84 \pm 5 \%)$.

Table 1 Mean $( \pm S D)$ of seminal parameters after collection in normal and poor quality ejaculates

\begin{tabular}{|c|c|c|}
\hline \multirow[t]{2}{*}{ Items } & $\begin{array}{l}\text { Normal semen } \\
\text { quality }\end{array}$ & $\begin{array}{l}\text { Poor semen } \\
\text { quality }\end{array}$ \\
\hline & Mean \pm SD & Mean $\pm S D$ \\
\hline Volume, mL & $7.8 \pm 2.5^{*}$ & $8.1 \pm 1^{*}$ \\
\hline Concentration, $\times 10^{6}$ sperm $/ \mathrm{mL}$ & $1192 \pm 203^{*}$ & $1048 \pm 171^{*}$ \\
\hline $\begin{array}{l}\text { Total sperm/ejaculate, } \times 10^{6} \\
\text { sperm } / \mathrm{mL}\end{array}$ & $10546 \pm 3226^{*}$ & $9432 \pm 1704^{*}$ \\
\hline Normal morphology, \% & $89.3 \pm 3.7^{*}$ & $64.8 \pm 11.6^{\S}$ \\
\hline Non-sperm cells, \% & $0.02 \pm 0.03^{*}$ & $2.9 \pm 0.8^{\S}$ \\
\hline $\mathrm{TM}, \%$ & $84 \pm 7^{*}$ & $73 \pm 4^{\S}$ \\
\hline PM, \% & $73 \pm 3^{*}$ & $59 \pm 2^{\S}$ \\
\hline$V A P, \mu \mathrm{m} / \mathrm{s}$ & $156.8 \pm 20.4^{*}$ & $140.5 \pm 11.7^{\S}$ \\
\hline$V S L, \mu m / s$ & $131.8 \pm 14.3^{*}$ & $119 \pm 8.9^{\S}$ \\
\hline$V C L, \mu \mathrm{m} / \mathrm{s}$ & $264.7 \pm 40.8^{*}$ & $223.8 \pm 17.6^{\S}$ \\
\hline $\mathrm{ALH}, \mu \mathrm{m}$ & $9 \pm 1.1^{*}$ & $8.7 \pm 0.3^{*}$ \\
\hline $\mathrm{BCF}, \mathrm{Hz}$ & $44.6 \pm 0.6^{*}$ & $42.9 \pm 1.7^{*}$ \\
\hline STR, $\%$ & $84 \pm 2^{*}$ & $85 \pm 2^{*}$ \\
\hline LIN, \% & $52 \pm 3^{*}$ & $50 \pm 1^{*}$ \\
\hline PI-, PSA-, \% & $80.4 \pm 10.1^{*}$ & $73.5 \pm 8.4^{*}$ \\
\hline $\mathrm{Pl}-, \mathrm{PSA}+, \%$ & $0.3 \pm 0.1^{*}$ & $0.5 \pm 0.2^{*}$ \\
\hline $\mathrm{PI}+, \mathrm{PSA}-, \%$ & $16.7 \pm 2.7^{*}$ & $21.9 \pm 3.1^{*}$ \\
\hline $\mathrm{PI}+, \mathrm{PSA}+, \%$ & $2.6 \pm 0.3^{*}$ & $3.1 \pm 0.5^{*}$ \\
\hline h-MMP, \% & $66.8 \pm 5.4^{*}$ & $62.6 \pm 6.9^{*}$ \\
\hline
\end{tabular}

TM Total motility, PM progressive motility, VAP average path velocity, VSL straight line velocity, $V C L$ curvilinear velocity, $A L H$ amplitude of lateral head displacement, BCF beat cross frequency, STR straightness, LIN linearity, PI-, PSAmembrane integrity and acrosome integrity, $P I-, P S A+$ membrane integrity and acrosome reaction, $P I+, P S A$ - membrane damage and acrosome integrity, $\mathrm{Pl}+$, $P S A+$ membrane damage and acrosome damage, $h-M M P$ spermatozoa with high mitochondrial membrane potential In the same row, values with different symbols in superscript $(* / \$)$ differ significantly $(P \leq 0.05)$
In all samples from normal bulls, sperm morphology was similar before and after centrifugation. The type of abnormality was found to be dependent upon the individual bull $(P \leq 0.01)$, but no differences were found in the percentage of each type before and after centrifugation in different treatments in normal samples (data not shown). Similarly, in all $\mathrm{N}$ samples the proportion of non-sperm cells was low, with values between 0.06 and $0.1 \%$. The proportion of sperm with abnormal morphology decreased significantly in P bulls treated with SLC and DLC, reaching similar values compared with samples before centrifugation in $\mathrm{N}$ bulls. No differences were found in sperm abnormal morphology in untreated and CC samples before and after centrifugation in P bulls. In these bulls, the percentage of non-sperm cells significantly decreased $(P \leq 0.01)$ after SLC (from $2.4 \pm 1.1 \%$ to $0.2 \pm 0.1 \%$ ) and DLC (from $3.1 \pm 1.6 \%$ to $0.08 \pm 0.06 \%$ ), but not in samples conventionally centrifuged and noncentrifuged. In all samples the morphology of spermatozoa after equilibration and after cryopreservation were similar for the corresponding treatment.

After centrifugation, SLC and DLC samples showed an increased sperm kinetic parameters in $\mathrm{P}$ bulls. As showed in Table 2, total motility and progressive motility were higher in SLC and DLC samples from P bulls compared with the value recorded in poor quality $\mathrm{NC}$ and CC sperm $(P \leq 0.05)$. Sperm velocities after centrifugation were similar to those in untreated spermatozoa. Conventional centrifugation seemed to increase the ALH parameter in both normal and poor quality samples $(P \leq 0.05)$, while BCF, STR and LIN were similar among treatments.

After equilibration for $3 \mathrm{~h}$ at $4{ }^{\circ} \mathrm{C}$, the proportion of total and progressive motility was similar to the values recorded for the corresponding treatment after centrifugation, such as ALH, BCF, STR, and LIN. On the other hand, VAP, VSL, and VCL were significantly lower in untreated $\mathrm{N}$ and $\mathrm{P}$ samples $(P \leq 0.05)$, but were similar in all centrifuged samples (data not shown). After cryopreservation, a significant decrease in sperm total and progressive motility was recorded in all samples compared with the respective pre-freezing samples. However, NC normal samples showed similar values compared with normal and poor quality samples centrifuged with SLC and DLC, while poor quality non-centrifuged samples and CC normal and poor quality samples showed lower values for total and progressive motility $(P \leq 0.05)$. A significant decrease in ALH was found after cryopreservation $(P \leq 0.05)$, with higher values in CC samples $(P \leq 0.05)$. The other kinetic parameters were similar in all treatments (Table 3).

The membrane integrity of spermatozoa centrifuged with SLC and DLC in both N and P bulls were comparable with the membrane integrity of $\mathrm{NC}$ samples in $\mathrm{N}$ 
Table 2 Mean $( \pm S D)$ of sperm characteristics after different centrifugation treatments in normal $(N)$ and poor $(P)$ quality ejaculates

\begin{tabular}{|c|c|c|c|c|c|c|c|c|}
\hline \multirow[t]{3}{*}{ Items } & \multicolumn{2}{|l|}{ SLC } & \multicolumn{2}{|l|}{$\mathrm{DLC}$} & \multicolumn{2}{|l|}{ CC } & \multicolumn{2}{|l|}{ NC } \\
\hline & $\mathrm{N}$ & $P$ & $\mathrm{~N}$ & $P$ & $\mathrm{~N}$ & $P$ & $\mathrm{~N}$ & $P$ \\
\hline & Mean \pm SD & Mean \pm SD & Mean \pm SD & Mean \pm SD & Mean \pm SD & Mean \pm SD & Mean \pm SD & Mean \pm SD \\
\hline Normal morphology, \% & $90.4 \pm 5.3^{*}$ & $91.2 \pm 4.4^{*}$ & $89.6 \pm 3.2^{*}$ & $91.3 \pm 3.7^{*}$ & $85.9 \pm 5.4^{*}$ & $63.7 \pm 6.4^{\S}$ & $87.9 \pm 4.6^{*}$ & $66.2 \pm 7.1^{\S}$ \\
\hline Non-sperm cells, $\%$ & $0.05 \pm 0.04^{*}$ & $0.07 \pm 0.04^{*}$ & $0.07 \pm 0.05^{*}$ & $0.05 \pm 0.03^{*}$ & $0.06 \pm 0.03^{*}$ & $2.8 \pm 1.1^{\S}$ & $0.03 \pm 0.02^{*}$ & $3.2 \pm 1.3^{\S}$ \\
\hline TM, \% & $91 \pm 6^{*}$ & $91 \pm 3^{*}$ & $91 \pm 5^{*}$ & $92 \pm 3^{*}$ & $85 \pm 6^{\S}$ & $86 \pm 6^{\S}$ & $84 \pm 7^{\S}$ & $83 \pm 4^{\S}$ \\
\hline PM, \% & $74 \pm 10^{*}$ & $79 \pm 4^{*}$ & $77 \pm 3^{*}$ & $81 \pm 1^{*}$ & $64 \pm 6^{\S}$ & $65 \pm 5^{\S}$ & $66 \pm 3^{\S}$ & $66 \pm 2^{\S}$ \\
\hline$V A P, \mu m / s$ & $153.2 \pm 10.9^{*}$ & $153.5 \pm 10.4^{*}$ & $150.1 \pm 10^{*}$ & $147.6 \pm 7.5^{*}$ & $152.7 \pm 13.9^{*}$ & $153.3 \pm 16.9^{*}$ & $156.8 \pm 20.4^{*}$ & $140.5 \pm 11.7^{\S}$ \\
\hline$V S L, \mu \mathrm{m} / \mathrm{s}$ & $127.2 \pm 9.4^{*}$ & $132.3 \pm 11.7^{*}$ & $129.2 \pm 8.6^{*}$ & $128.4 \pm 7.7^{*}$ & $126.1 \pm 9.9^{*}$ & $126.7 \pm 16.5^{*}$ & $131.8 \pm 14.3^{*}$ & $119 \pm 8.9^{\S}$ \\
\hline$V C L, \mu \mathrm{m} / \mathrm{s}$ & $256.6 \pm 35.9^{* \S}$ & $263.4 \pm 18.5^{\S}$ & $252.4 \pm 25.8^{*}$ & $255.9 \pm 14.1^{*}$ & $261.6 \pm 25.7^{*}$ & $271.1 \pm 19.3^{\S}$ & $264.7 \pm 40.8^{\S}$ & $243.8 \pm 17.6^{*}$ \\
\hline $\mathrm{ALH}, \mu \mathrm{m}$ & $8.9 \pm 1.7^{*}$ & $8.7 \pm 0.7^{*}$ & $8.6 \pm 1.2^{*}$ & $8.8 \pm 0.5^{*}$ & $9.3 \pm 0.8^{\S}$ & $9.6 \pm 0.3^{\S}$ & $8.8 \pm 1.1^{*}$ & $8.7 \pm 0.3^{*}$ \\
\hline $\mathrm{BCF}, \mathrm{Hz}$ & $44.3 \pm 3.3^{*}$ & $45.2 \pm 3^{*}$ & $45.2 \pm 1.9^{*}$ & $44.7 \pm 1.4^{*}$ & $43.8 \pm 1.9^{*}$ & $42.7 \pm 2.6^{*}$ & $44.6 \pm 1.6^{*}$ & $42.9 \pm 1.7^{*}$ \\
\hline STR, $\%$ & $83 \pm 3^{*}$ & $86 \pm 2^{*}$ & $86 \pm 4^{*}$ & $87 \pm 2^{*}$ & $83 \pm 2^{*}$ & $82 \pm 3^{*}$ & $84 \pm 2^{*}$ & $85 \pm 2^{*}$ \\
\hline LIN, \% & $51 \pm 3^{*}$ & $53 \pm 2^{*}$ & $53 \pm 3^{*}$ & $53 \pm 2^{*}$ & $51 \pm 2^{*}$ & $50 \pm 2^{*}$ & $52 \pm 1.5^{*}$ & $51 \pm 2^{*}$ \\
\hline PI-, PSA-, \% & $86.9 \pm 5.4^{*}$ & $85.1 \pm 8.3^{*}$ & $83.7 \pm 7.3^{*}$ & $84.5 \pm 3^{*}$ & $75.5 \pm 10.4^{\S}$ & $72.3 \pm 4.7^{\S}$ & $82.4 \pm 7.27^{*}$ & $71.3 \pm 10.3^{\S}$ \\
\hline $\mathrm{Pl}-, \mathrm{PSA}+, \%$ & $0.5 \pm 0.2^{*}$ & $0.4 \pm 0.2^{*}$ & $0.3 \pm 0.2^{*}$ & $0.4 \pm 0.3^{*}$ & $0.6 \pm 0.2^{*}$ & $0.7 \pm 0.3^{*}$ & $0.5 \pm 0.3^{*}$ & $0.7 \pm 0.4^{*}$ \\
\hline $\mathrm{PI}+, \mathrm{PSA}-, \%$ & $10.3 \pm 0.9^{*}$ & $12 \pm 1.1^{*}$ & $13.8 \pm 1.2^{*}$ & $12.6 \pm 0.9^{*}$ & $20.8 \pm 1.7^{\S}$ & $24 \pm 1.4^{\S}$ & $14.9 \pm 1.1^{*}$ & $24.9 \pm 1.3^{\S}$ \\
\hline $\mathrm{PI}+, \mathrm{PSA}+, \%$ & $2.3 \pm 0.4^{*}$ & $2.5 \pm 0.3^{*}$ & $2.2 \pm 0.3^{*}$ & $2.5 \pm 0.2^{*}$ & $3.1 \pm 0.4^{*}$ & $3 \pm 0.3^{*}$ & $2.2 \pm 0.3^{*}$ & $3.1 \pm 0.4^{*}$ \\
\hline h-MMP, \% & $53.6 \pm 4.2^{*}$ & $52.9 \pm 3.8^{*}$ & $54.2 \pm 4.6^{*}$ & $53.1 \pm 4.1^{*}$ & $52.4 \pm 3.7^{*}$ & $51.9 \pm 3.2^{*}$ & $45.8 \pm 3.2^{\S}$ & $41.8 \pm 6.4^{\S}$ \\
\hline
\end{tabular}

$T M$ Total motility, PM progressive motility, VAP average path velocity, VSL straight line velocity, VCL curvilinear velocity, $A L H$ amplitude of lateral head displacement, $B C F$ beat cross frequency, STR straightness, LIN linearity, Pl-, PSA-membrane integrity and acrosome integrity, Pl-, PSA+ membrane integrity and acrosome damage, $P I+, P S A-$ membrane damage and acrosome integrity, $P I+, P S A+$ membrane damage and acrosome damage, $h-M M P$ spermatozoa with high mitochondrial membrane potential In the same row, values with different symbols in superscript $(* / 5)$ differ significantly $(P \leq 0.05)$

Table 3 Mean ( \pm SD) of cryopreserved sperm characteristics different treatments in normal $(N)$ and poor $(P)$ quality ejaculates

\begin{tabular}{|c|c|c|c|c|c|c|c|c|}
\hline \multirow[t]{3}{*}{ Items } & \multicolumn{2}{|l|}{$\mathrm{SLC}$} & \multicolumn{2}{|l|}{$\mathrm{DLC}$} & \multicolumn{2}{|l|}{ CC } & \multicolumn{2}{|l|}{$\mathrm{NC}$} \\
\hline & $\mathrm{N}$ & $P$ & $\mathrm{~N}$ & $P$ & $\mathrm{~N}$ & $P$ & $\mathrm{~N}$ & $P$ \\
\hline & Mean \pm SD & Mean \pm SD & Mean \pm SD & Mean \pm SD & Mean \pm SD & Mean \pm SD & Mean \pm SD & Mean \pm SD \\
\hline $\mathrm{TM}, \%$ & $55.5 \pm 5.2^{*}$ & $54.3 \pm 7.2^{*}$ & $55.8 \pm 8.6^{*}$ & $56.8 \pm 5.6^{*}$ & $44.5 \pm 15.5^{\S}$ & $45 \pm 11.5^{\S}$ & $56.7 \pm 6.3^{*}$ & $45.3 \pm 9.1^{\S}$ \\
\hline $\mathrm{PM}, \%$ & $42.7 \pm 6.4^{*}$ & $43 \pm 6.5^{*}$ & $41.5 \pm 5.5^{*}$ & $43.8 \pm 5.1^{*}$ & $25.8 \pm 13.4^{\S}$ & $28 \pm 7.2^{\S}$ & $42.1 \pm 6.7^{*}$ & $34 \pm 9.4^{* \S}$ \\
\hline$V A P, \mu m / s$ & $124.8 \pm 11.5^{*}$ & $122.3 \pm 12.1^{* \$}$ & $127.8 \pm 16.1^{*}$ & $128.9 \pm 18.4^{*}$ & $115.8 \pm 17.2^{\S}$ & $119 \pm 10.9^{* \S}$ & $127.4 \pm 13.4^{*}$ & $116.7 \pm 7.6^{\S}$ \\
\hline$V S L, \mu \mathrm{m} / \mathrm{s}$ & $109.6 \pm 15^{*}$ & $101.7 \pm 10.1^{*}$ & $108.7 \pm 15.7^{*}$ & $105.3 \pm 18.7^{*}$ & $100.3 \pm 18.1^{*}$ & $108.1 \pm 10.3^{*}$ & $110 \pm 14.5^{*}$ & $100.6 \pm 9^{*}$ \\
\hline$V C L, \mu \mathrm{m} / \mathrm{s}$ & $193 \pm 10^{* \S}$ & $179.1 \pm 17.4^{*}$ & $181.1 \pm 27.5^{*}$ & $177.3 \pm 35.7^{*}$ & $202.8 \pm 19.4^{* \S}$ & $225.5 \pm 15.7^{\S}$ & $212 \pm 17.9^{* \S}$ & $164.9 \pm 6.2^{*}$ \\
\hline $\mathrm{ALH}, \mu \mathrm{m}$ & $7.4 \pm 0.7^{*}$ & $6.9 \pm 0.7^{*}$ & $7.7 \pm 0.9^{*}$ & $7.1 \pm 1.6^{*}$ & $8.3 \pm 0.5^{\S}$ & $8.6 \pm 1.2^{\S}$ & $7.7 \pm 0.7^{*}$ & $7.5 \pm 0.7^{*}$ \\
\hline $\mathrm{BCF}, \mathrm{Hz}$ & $38.6 \pm 2.4^{*}$ & $37.1 \pm 1.4^{*}$ & $33.8 \pm 4.1^{*}$ & $33.4 \pm 4.4^{*}$ & $36.8 \pm 1.4^{*}$ & $35.7 \pm 1.2^{*}$ & $38.7 \pm 3.1^{*}$ & $35.8 \pm 3.2^{*}$ \\
\hline STR, $\%$ & $83.8 \pm 4.4^{*}$ & $83.8 \pm 2.9^{*}$ & $80.3 \pm 1.9^{*}$ & $81.5 \pm 2.5^{*}$ & $81.5 \pm 4.4^{*}$ & $82 \pm 1^{*}$ & $83.8 \pm 2.8^{*}$ & $84.8 \pm 3.2^{*}$ \\
\hline LIN, \% & $51.7 \pm 5.9^{*}$ & $49.8 \pm 3.8^{*}$ & $45.7 \pm 2.7^{*}$ & $48 \pm 2.2^{*}$ & $48 \pm 5.7^{*}$ & $49 \pm 1.7^{*}$ & $51.7 \pm 3.4^{*}$ & $52.5 \pm 3.7^{*}$ \\
\hline PI-, PSA-, \% & $51.3 \pm 5.8^{*}$ & $50.8 \pm 8.4^{*}$ & $52.5 \pm 7.9^{*}$ & $51 \pm 7.2^{*}$ & $42.6 \pm 5^{\S}$ & $38.9 \pm 6.5^{\S}$ & $54.3 \pm 8.1^{*}$ & $39.4 \pm 6.8^{\S}$ \\
\hline Pl-, PSA+, \% & $8.3 \pm 2^{*}$ & $8.2 \pm 1.2^{*}$ & $6.6 \pm 1.4^{*}$ & $7.3 \pm 1.8^{*}$ & $9.3 \pm 2.4^{*}$ & $12.2 \pm 4.5^{*}$ & $8 \pm 2^{*}$ & $8.6 \pm 1.1^{*}$ \\
\hline $\mathrm{Pl}+, \mathrm{PSA}-, \%$ & $26.1 \pm 5.8^{* \S}$ & $27.3 \pm 5.9^{* \S}$ & $22.6 \pm 2.3^{*}$ & $24.5 \pm 5.5^{* \S}$ & $27.4 \pm 6.1^{* \S}$ & $27.1 \pm 4.6^{* \S}$ & $22.3 \pm 3.3^{*}$ & $33.5 \pm 6.8^{\S}$ \\
\hline $\mathrm{Pl}+, \mathrm{PSA}+, \%$ & $14.3 \pm 2.6^{*}$ & $13.7 \pm 3.9^{*}$ & $18.3 \pm 2.6^{*}$ & $17.2 \pm 3.8^{*}$ & $20.7 \pm 5.2^{*}$ & $21.9 \pm 6.9^{*}$ & $15.4 \pm 3.2^{*}$ & $18.5 \pm 6.3^{*}$ \\
\hline h-MMP, \% & $34.1 \pm 7.1^{*}$ & $35.7 \pm 5.4^{*}$ & $38.6 \pm 10.9^{*}$ & $31.6 \pm 11.4^{*}$ & $33.7 \pm 3.4^{*}$ & $30.9 \pm 17.2^{*}$ & $35.9 \pm 8.2^{*}$ & $32.6 \pm 9.2^{*}$ \\
\hline
\end{tabular}

TM Total motility, PM progressive motility, VAP average path velocity, VSL straight line velocity, VCL curvilinear velocity, $A L H$ amplitude of lateral head displacement, $B C F$ beat cross frequency, STR straightness, LIN linearity, Pl-, PSA- membrane integrity and acrosome integrity, Pl-, PSA+ membrane integrity and acrosome reaction, PI+, PSAmembrane damage and acrosome integrity, $\mathrm{Pl}+, P S A+$ membrane damage and acrosome damage, $h-M M P$ spermatozoa with high mitochondrial membrane potential In the same row, values with different symbols in superscript $\left(* /^{5}\right)$ differ significantly $(P \leq 0.05)$ 
bulls. On the other hand, CC significantly decreased sperm membrane integrity in both $\mathrm{N}$ and $\mathrm{P}$ bulls, and the data recorded were similar to that in NC spermatozoa of P bulls (Table 2). A similar trend was found after equilibration. Surprisingly, sperm membrane integrity in samples after centrifugation or after equilibration was lower than sperm total motility in all treatments, suggesting that some spermatozoa with sub-lethal membrane damage could have flagellar beat. Cryopreservation dramatically impacted on sperm membrane integrity, since a significant decrease in sperm with membrane integrity was recorded in NC such as in SLC, DLC, and CC samples (Table 3). Sperm with reacted acrosomes were similar in all treatments and increased significantly only after cryopreservation $(P \leq 0.05)$.

A significant increase in sperm with high MMP was found in all centrifuged samples (SLC, DLC, and CC) compared with the NC samples soon after centrifugation, but the differences become not significant after cryopreservation. At this stage, spermatozoa of $\mathrm{N}$ bulls in NC samples showed similar values compared with SLC and DLC spermatozoa in both N and P bulls, and with CC spermatozoa in $\mathrm{N}$ bulls. On the other hand, a significantly lower proportion of spermatozoa with high MMP was found in NC and CC samples of P bulls $(P \leq 0.05)$ (Table 3$)$.

\section{Discussion}

In several cases the semen quality of high genetic bulls could be transiently or permanently decreased, resulting in the elimination of the batch from the cryopreservation procedure. This could result in a relevant economic loss for the artificial insemination centers. In a previous study gradient centrifugation was proposed as a method to select sperm subpopulation of normal size in a polymorphic bull [26]. In the present study the single and the double layer centrifugation procedure, by the use of iodixanol, significantly improved the quality of the poor quality bull samples.

In our study, sperm morphology significantly improved after SLC and DLC in poor quality samples. The abnormal spermatozoa were considered more buoyant compared to normal sperm [27], and it was apparent that the density of spermatozoa increased with maturation [28]. Although the latter parameter was not specifically considered in this study, the increase in the DNA integrity reported in several papers on spermatozoa collected in the pellet after SLC confirmed this hypothesis, in humans $[29,30]$ and animals $[16,31-33]$. On the other hand, in samples of normal quality, sperm morphology after both SLC and DLC was similar compared to that of untreated or CC samples. The improvement of sperm morphology after gradient centrifugation is debated in literature, with studies where the sperm with normal morphology increases [32], and others in which this parameter was similar to the untreated samples $[11,16]$. As in the latter cases, our results suggest that when the sperm morphological quality is high, the improvement is negligible. Single and double layer centrifugation seemed to remove also other cells and debris present in the ejaculate. In the samples with poor quality, epithelial cells, white blood cells, or cell debris, were reduced after centrifugation. Previous studies in horses showed that SLC or density gradient centrifugation [11] decreased the number of non-sperm cells in the pellet, and that this could reduce the formation of myeloperoxidase in the post-thaw samples [34]. This improvement is not reported in the cushioned centrifugation, in which both spermatozoa and cell debris are usually collected in the pellet $[35,36]$.

In poor quality samples, sperm kinetic characteristics significantly increased after SLC and DLC, with values similar to those reported in normal quality samples. Total and progressive motility increased after SLC and DLC compared with untreated and CC samples in all normal samples; this was evident also after equilibration. On the other hand, negligible effects were found on the other kinetic parameters in normal bulls, with the exception that ALH increased in CC samples, and the difference became significant after cryopreservation. Our findings were different to those reported in a previous study, in which total and progressive motility were found to be similar in samples untreated and centrifuged by single layer (SLC): sperm straightness, linearity, and beat cross frequency were significantly higher in SLC, whereas velocity of the average path, curvilinear velocity and amplitude of lateral head deviation all significantly decreased [37]. These differences in the kinematic aspect could be due to the treatment of the samples before analysis. In that study, bull semen was transported overnight at $4{ }^{\circ} \mathrm{C}$ before centrifugation and evaluation, and this could affect the results. Furthermore, a different device was used for the analysis of sperm kinematics, which could explain the difference in values reported in our trials. Our previous study has clarified that the technical characteristics of the computer assisted sperm analyzer could affect the results [23].

Sperm mitochondrial activity increased in poor quality samples after centrifugation, and this was maintained also during equilibration and cryopreservation. This could be related to the sperm kinetic parameters, since a similar trend was found for these characteristics. On the other hand, no differences were found in samples before and after centrifugation in normal bulls, in contrast with previous findings that showed a significant increase in mitochondrial membrane potential (from 56 to $83 \%$ ) after SLC [16]. 
The bull samples treated by SLC and DLC with iodixanol showed a similar sperm quality after cryopreservation compared with the untreated samples and the CC samples. This was in contrast with studies in the stallion $[15,38]$ and in the boar [39], in which the removal of seminal plasma improve the sperm resistance to the cryoinjury. Furthermore, Heutelbeck et al. [12] hypothesized that the higher sperm membrane integrity after cryopreservation in samples processed using a two-layer iodixanol density centrifugation was due to the cryoprotective effect of the iodixanol [40]. This hypothesis was unconfirmed by our data, since the both samples treated with iodixanol (SLC and DLC) showed similar parameters after thawing compared with untreated samples. A possible explanation is that the concentration of the iodixanol could be too low to give a cryoprotective effect.

In samples centrifuged using the conventional procedure (CC samples) total and progressive motility decreased significantly in both normal and poor quality samples after cryopreservation, but this effect was not present soon after centrifugation or equilibration and apparently did not involve the other kinetic properties. Conventional centrifugation results in a sperm pellet formation at the bottom of the centrifugation tube, and this is believed to have a negative effect on spermatozoa [13-15]. These results suggested that latent damage, not visible during liquid storage, could be present as a consequence of the centrifugation stress, but this effect was not elicited during liquid storage, and only after cryopreservation. However, specific trials to verify this hypothesis should be performed.

One of the problems related to the applicability of density centrifugation was the loss of spermatozoa. In the present study the recovery rate recorded in samples of normal quality centrifuged using SLC (56 \%) and DLC (44. \%) was significantly lower compared with the CC method (84 \%). The data reported here were comparable with those reported using a DLC in the stallion [11], in which the iodixanol density gradient centrifugation allowed a recovery rate of $33 \%$, while the ordinary centrifugation reached a $74 \%$ recovery rate. Several studies in different species showed that SLC, by the use of silane-coated silica colloid, resulted in a significant loss of spermatozoa, with a yield rate ranging from 30 to $65 \%$ [32] in horses, and a $50 \%$ in bulls [9]. The higher recovery rate reported in this study compared with data reported in the SLC using silane-coated silica colloid could be the result of the variations in g-force used for the centrifugation. The centrifugation speed could be considered one of the most important factors affecting sperm recovery during CC [15], and this could be applied also to SLC. The density centrifugation was in all cases related to a reduced amount of spermatozoa recovered after centrifugation. Thus, it was concluded that this procedure may be inapplicable in practice when semen of normal quality should be handled. However, it could be considered when the sperm characteristics are of greater importance than the total amount of spermatozoa recovered after density centrifugation [12]. This is the case of our study, in which sperm of low quality were treated by SLC and DLC. In both treatments, sperm characteristics significantly improved.

The increase in recovery rate could be obtained by the increase in the centrifugation force. Cushioned centrifugation was proposed to increase the recovery rate of spermatozoa by higher gravitational force avoiding harmful effects on semen [41, 42]. During cushioned centrifugation, the use of a dense cushion, mainly iodixanol, in the bottom of the centrifugation tube was used to avoid the excessive compaction of the sperm pellet during high speed centrifugation [43]. The data reported in literature showed that a centrifugation speed exceeding $1,800 \times \mathrm{g}$ could be deleterious for equine sperm motility [44], while centrifugation at $600 \times \mathrm{g}$ [45], $900 \times \mathrm{g}$ [44], and $1,000 \times \mathrm{g}$ [43] had a negligible effect on equine sperm characteristics. However, the data reported in the present study showed that the presence of a cushion (DLC) did not improve the seminal quality either after centrifugation, or after equilibration or cryopreservation, compared with SLC.

\section{Conclusions}

In this study the effects of single layer centrifugation and double layer centrifugation on bull sperm characteristics after cryopreservation in normal and poor quality ejaculates were studied. In bulls with normal semen characteristics, no significant effects were found in samples before and after SLC and DLC compared with untreated samples. Single layer centrifugation and DLC seemed to be useful tools to improve sperm characteristics of poor quality semen in bulls of high genetic value, where the sperm characteristics after both these procedures were comparable to those of normal samples. Thus, these procedures could be applicable in practice when the quality of the spermatozoa recovered is of greater importance than the total amount of spermatozoa.

\section{Abbreviations \\ Al: artificial insemination; ALH: amplitude of lateral head displacement; BCF: beat cross frequency; CASA: computer assisted sperm analyzer; CC: conventional centrifugation; DLC: double layer centrifugation; FITC- PSA: FITC-conjugated agglutinin derived from pisum sativum; JC-1: 5,5',6,6'- tetrachloro-1,1',3,3'-tetraethyl-benzimidazolylcarbocyanine chloride; LIN: linearity; MMP: Mitochondrial membrane potential; N: normal quality semen; NC: non-centrifuged; P: poor quality semen; PI: propidium iodide; PM: progressive motility; ROS: reactive oxygen species; SLC: single layer centrifugation; STR: straightness; TALP: tyrode's medium; TM: total motility; VAP: average path velocity; VCL: curvilinear velocity; VSL: straight line velocity.}

Competing interests

The authors declare that they have no competing interests. 


\section{Authors' contributions}

GA and CoA carried out the experiment design and trial, data interpretation and manuscript writing. CoA performed the statistics. WL, RD and BG helped with data collection. CA participated in discussion. All authors read and approved the final manuscript.

\section{Acknowledgments}

Alessia Gloria is a DVM postdoctoral fellow supported by a grant from Regione Abruzzo (P.O. FSE Abruzzo 2007 - 2013, Action 4, Type 4.1). The authors are grateful to the Provincial Breeders Federation of Trento, especially Dr. Claudio Valorz, for their support. The authors are also grateful to the Superbrown Consortium Bz/Tn and the "Alpenseme" Al Center (Ton, Trento - Italy) staff for their cooperation.

\section{Author details}

${ }^{1}$ Faculty of Veterinary Medicine, University of Teramo, Località Piano D'Accio, 64100 Teramo, Italy. ${ }^{2}$ Provincial Breeders Federation of Trento, Via delle Bettine 40, 38121 Trento, Italy. ${ }^{3}$ Associazione Regionale Allevatori d'Abruzzo (ARA), S.S. 17 Est loc., Onna, L'Aquila, Italy.

\section{Received: 8 November 2015 Accepted: 24 April 2016 Published online: 05 May 2016}

\section{References}

1. Morrell JM, Rodriguez-Martinez H. Biometric techniques for improving sperm quality in animal breeding: a review. Open Androl. 2009;1:1-9.

2. Chong AP, Walters CA, Weinried SA. The neglected laboratory test. The semen analysis. J Androl. 1983;4:280-2.

3. Vantman D, Koukoulis G, Dennison L, Zinaman M, Sherins RJ. Computerassisted semen analysis: evaluation of method and assessment of the influence of sperm concentration on linear velocity determination. Fertil Steril. 1988:49:510-5.

4. Comhaire FH, Huysse S, Hinting A, Vermeulen L, Schoonjans F. Objective semen analysis: has the target been reached? Hum Reprod. 1992;7:237-41.

5. Rijsselaere T, Van Soom A, Maes D, de Kruif A. Use of the sperm quality analyzer (SQA II-C) for the assessment of dog sperm quality. Reprod Domest Anim. 2002;37:158-63.

6. World Health Organization (WHO). Laboratory manual for the examination of human semen and sperm-cervical mucus interaction, 4th edn. Cambridge: Cambridge University Press; 1999. p. 128.

7. Rodriguez-Martinez H, Larsson B, Pertoft H. Evaluation of sperm damage and techniques for sperm clean-up. Reprod Fertil Dev. 1997;9:297-308.

8. Hallap T, Haard M, Jaakma U, Larsson B, Rodriguez-Martinez H. Does cleansing of frozen-thawed bull semen before assessment provide samples that relate better to potential fertility? Theriogenology. 2004;62:702-13.

9. Thys M, Vandaele L, Morrell JM, Mestach J, Van Soom A, Hoogewijs M, et al. In vitro fertilizing capacity of frozen-thawed bull spermatozoa selected by single-layer (glycidoxypropyltrimethoxysilane) silane-coated silica colloidal centrifugation. Reprod Domest Anim. 2009:44:390-4.

10. Morrell JM. Update on semen technologies for animal breeding. Reprod Domest Anim. 2006:41:63-7.

11. Stuhtmann G, Oldenhof $H$, Peters P, Klewitz J, Martinsson G, Sieme H. lodixanol density gradient centrifugation for selecting stallion sperm for cold storage and cryopreservation. Anim Reprod Sci. 2012;133:184-90.

12. Heutelbeck A, Oldenhof H, Rohn K, Martinsson G, Morrell JM, Sieme H. Use of density centrifugation for delayed cryopreservation of stallion sperm: perform sperm selection directly after collection or after storage? Reprod Domest Anim. 2015:50:76-83.

13. Pickett BW, Sullivan JJ, Byers WW, Pace MM, Remmenga EE. Effect of centrifugation and seminal plasma on motility and fertility of stallion and bull spermatozoa. Fertil Steril. 1975;26:167-74.

14. Jasko DJ, Lein $\mathrm{DH}$, Foote $\mathrm{RH}$. The repeatability and effect of season on seminal characteristics and computer-aided sperm analysis in the stallion. Theriogenology. 1991;35:317-27.

15. Hoogewijs M, Rijsselaere T, De Vliegher S, Vanhaesebrouck E, De Schauwer C, Govaere J, et al. Influence of different centrifugation protocols on equine semen preservation. Theriogenology. 2010;74:118-26.

16. Goodla L, Morrell JM, Yusnizar Y, Stålhammar H, Johannisson A. Quality of bull spermatozoa after preparation by single-layer centrifugation. J Dairy Sci. 2014;97:2204-12
17. Hancock JL. The morphology of boar spermatozoa. J R Microsc Soc. 1957; 76:84-97.

18. Contri A, Gloria A, Robbe D, Valorz C, Wegher L, Carluccio A. Kinematic study on the effect of pH on bull sperm function. Anim Reprod Sci. 2013; 136:252-9.

19. Parrish JJ, Susko-Parrish J, Winer MA, First NL. Capacitation of bovine sperm by heparin. Biol Reprod. 1988;38:1171-80.

20. Muino R, Rivera MM, Rigau T, Rodriguez-Gil JE, Pena Al. Effect of different thawing rates on post-thaw sperm viability, kinematic parameters and motile sperm subpopulations structure of bull semen. Anim Reprod Sci. 2008:109:50-64.

21. Barth AD, Oko RJ. Abnormal morphology of bovine spermatozoa. Ames: lowa State University Press; 1989.

22. Gloria A, Carluccio A, Contri A, Wegher L, Valorz C, Robbe D. The effect of the chamber on kinetic results in cryopreserved bull spermatozoa. Andrology. 2013;1:879-85.

23. Contri A, Valorz C, Faustini M, Wegher L, Carluccio A. Effect of semen preparation on CASA motility results in cryopreserved bull spermatozoa. Theriogenology. 2010;74:424-35.

24. Bollwein $\mathrm{H}$, Fuchs I, Koess C. Interrelationship between plasma membrane integrity, mitochondrial membrane potential and DNA fragmentation in cryopreserved bovine spermatozoa. Reprod Dom Anim. 2008;43:189-95.

25. Garner DL, Thomas CA. Organelle-specific probe JC-1 identifies membrane potential differences in the mitochondrial function of bovine sperm. Mol Reprod Dev. 1999;53:222-9.

26. Morrell JM, Rodriguez-Martinez H, Andersson M. Colloid centrifugation selects normal spermatozoa from polymorphic bull ejaculates: a case study. Reprod Domest Anim. 2014:49:281-4.

27. Edmond AJ, Brinsko SP, Love CC, Blanchard TL, Teague SR, Varner DD. Effect of centrifugal fractionation protocols on quality and recovery rate of equine sperm. Theriogenology. 2012;77:959-66.

28. Oshio S. Apparent densities of spermatozoa of various mammalian species. Gamete Res. 1988;20:159-64.

29. Tomlinson MJ, Moffatt O, Manicardi GC, Bizzaro D, Afnan M, Sakkas D. Interrelationships between seminal parameters and sperm nuclear DNA damage before and after density gradient centrifugation: implications for assisted conception. Hum Reprod. 2001;16:2160-5.

30. Brahem S, Mehdi M, Elghezal H, Saad A. Semen processing by density gradient centrifugation is useful in selecting sperm with higher doublestrand DNA integrity. Andrologia. 2011:43:196-202.

31. Morrell JM, Rodriguez-Martinez H, Johannisson A. Single layer centrifugation of stallion spermatozoa consistently selects the most robust spermatozoa from the rest of the ejaculate in a large sample size. Equine Vet J. 2010;42: 579-85.

32. Morrell JM, Johannisson A, Dalin AM, Rodriguez-Martinez H. Morphology and chromatin integrity of stallion spermatozoa prepared by density gradient and single layer centrifugation through silica colloids. Reprod Domest Anim. 2009;44:512-7

33. Johannisson A, Morrell JM, Thorén J, Jönsson M, Dalin AM, RodriguezMartinez H. Colloidal centrifugation with Androcoll-E prolongs stallion sperm motility, viability and chromatin integrity. Anim Reprod Sci. 2009;116: 119-28.

34. Ponthier J, Teague SR, Franck TY, de la Rebière G, Serteyn DD, Brinsko SP, et al. Effect of non-sperm cells removal with single-layer colloidal centrifugation on myeloperoxidase concentration in post-thaw equine semen. Theriogenology. 2013;80:1082-7.

35. Sieme $H$, Martinsson $G$, Rauterberg $H$, Walter $K$, Aurich $C$, Petzoldt $R$, et al. Application of techniques for sperm selection in fresh and frozen-thawed stallion semen. Reprod Domest Anim. 2003;38:134-40.

36. Mousset-Simeon N, Rives N, Masse L, Chevallier F, Mace B. Comparison of six density gradient media for selection of cryopreserved donor spermatozoa. J Androl. 2004;25:881-4

37. Yulnawati Y, Abraham MC, Laskowski D, Johannisson A, Morrell JM. Changes in bull sperm kinematics after single layer centrifugation. Reprod Domest Anim. 2014;49:954-6.

38. Hoogewijs M, Morrell J, Van Soom A, Govaere J, Johannisson A, Piepers S, et al. Sperm selection using single layer centrifugation prior to cryopreservation can increase thawed sperm quality in stallions. Equine Vet J. 2011;40:35-41.

39. Martinez-Alborcia MJ, Morrell JM, Gil MA, Barranco I, Maside C, Alkmin DV, et al. Suitability and effectiveness of single layer centrifugation using 
Androcoll-P in the cryopreservation protocol for boar spermatozoa. Anim Reprod Sci. 2013;140:173-9.

40. Saragusty J, Gacitua H, Rozenboim I, Arav A. Protective effects of iodixanol during bovine sperm cryopreservation. Theriogenology. 2009;71:1425-32.

41. Ecot P, Decuadro-Hansen G, Delhomme G, Vidament M. Evaluation of a cushioned centrifugation technique for processing equine semen for freezing. Anim Reprod Sci. 2005;89:245-8.

42. Knop K, Hoffmann N, Rath D, Sieme H. Effects of cushioned centrifugation technique on sperm recovery and sperm quality in stallions with good and poor semen freezability. Anim Reprod Sci. 2005;89:294-7.

43. Bliss SB, Voge JL, Hayden SS, Teague SR, Brinsko SP, Love CC, et al. The impact of cushioned centrifugation protocols on semen quality of stallions. Theriogenology. 2012;77:1232-9.

44. Len JA, Beehan DP, Lyle SK, Eilts BE. Cushioned versus noncushioned centrifugation: sperm recovery rate and integrity. Theriogenology. 2013;80: 648-53.

45. Waite JA, Love CC, Brinsko SP, Teague SR, Salazar Jr JL, Mancill SS, et al. Factors impacting equine sperm recovery rate and quality following cushioned centrifugation. Theriogenology. 2008;70:704-14.

Submit your next manuscript to BioMed Central and we will help you at every step:

- We accept pre-submission inquiries

- Our selector tool helps you to find the most relevant journal

- We provide round the clock customer support

- Convenient online submission

- Thorough peer review

- Inclusion in PubMed and all major indexing services

- Maximum visibility for your research

Submit your manuscript at www.biomedcentral.com/submit
Biomed Central 